\title{
Imaging radar observations of Farley Buneman waves during the JOULE II experiment
}

\author{
D. L. Hysell ${ }^{1}$, G. Michhue ${ }^{1}$, M. F. Larsen ${ }^{2}$, R. Pfaff ${ }^{3}$, M. Nicolls ${ }^{4}$, C. Heinselman ${ }^{4}$, and H. Bahcivan ${ }^{4}$ \\ ${ }^{1}$ Earth and Atmospheric Sciences, Cornell University, Ithaca, NY, USA \\ ${ }^{2}$ Department of Physics, Clemson University, Clemson, SC, USA \\ ${ }^{3}$ NASA Goddard Space Flight Center, Greenbelt, MD, USA \\ ${ }^{4}$ SRI International, Menlo Park, CA, USA
}

Received: 23 January 2008 - Revised: 18 April 2008 - Accepted: 20 May 2008 - Published: 3 July 2008

\begin{abstract}
Vector electric fields and associated $\boldsymbol{E} \times \boldsymbol{B}$ drifts measured by a sounding rocket in the auroral zone during the NASA JOULE II experiment in January 2007, are compared with coherent scatter spectra measured by a $30 \mathrm{MHz}$ radar imager in a common volume. Radar imaging permits precise collocation of the spectra with the background electric field. The Doppler shifts and spectral widths appear to be governed by the cosine and sine of the convection flow angle, respectively, and also proportional to the presumptive ion acoustic speed. The neutral wind also contributes to the Doppler shifts. These findings are consistent with those from the JOULE I experiment and also with recent numerical simulations of Farley Buneman waves and instabilities carried out by Oppenheim et al. (2008). Simple linear analysis of the waves offers some insights into the spectral moments. A formula relating the spectral width to the flow angle, ion acoustic speed, and other ionospheric parameters is derived.
\end{abstract}

Keywords. Ionosphere (Auroral ionosphere; Ionospheric irregularities; Plasma waves and instabilities)

\section{Introduction}

A sounding rocket experiment was conducted from the Poker Flat Research Range in January of 2007 to investigate fine structure in the auroral convection electric field and the contribution this makes to the overall rate of Joule heating during substorms. Since Joule heating depends on the electric field quadratically, spatio-temporal averaging in the measurement of the former leads to some underestimation of the latter (Codrescu et al., 1995). To assess the importance of the error, electric fields were measured with instruments sensitive to a wide range of spatial and temporal scales. Two instrumented sounding rockets were used to measure electric fields

Correspondence to: D. L. Hysell

(dlh37@cornell.edu) down to very small (sub-meter) scale sizes. On the opposite end of the spectrum, the Poker Flat Incoherent Scatter Radar (PFISR) was used to estimate the regional convection pattern on much coarser (tens of $\mathrm{km}$ ) spatial scales. Since it is the electric field in the neutral frame of reference that determines Joule heating, chemical releases were also made for neutral wind profile measurements.

To investigate phenomena in the intermediate scale-size regime, a coherent scatter radar was used to measure backscatter from auroral E-region field-aligned irregularities (FAIs) in the rocket launch zone. These irregularities are caused by Farley Buneman instabilities which occur when the electric field exceeds about $20 \mathrm{mV} / \mathrm{m}$ (see reviews by Haldoupis, 1989; Sahr and Fejer, 1996; Moorcroft, 2002). The radar uses spaced antenna techniques and statistical inverse theory to produces two-dimensional images of the irregularities with a spatial resolution a few kilometers in each direction and a temporal resolution of a few seconds.

Coherent scatter spectra from E-region auroral irregularities are clearly affected by the magnitude and direction of the convection electric field, but the relationship is not so direct as in the case of F-region FAIs, and the prospects of inferring electric fields from the radar data are unresolved (St.Maurice et al., 1981; Nielsen and Schlegel, 1985; Robinson, 1986; Foster and Erickson, 2000; Milikh and Dimant, 2002; Nielsen et al., 2002; Uspensky et al., 2003, 2004; Makarevich et al., 2006). Using imaging radar and rocket data from the JOULE I experiment, a precursor to this one, Bahcivan et al. (2005) found a cosine relationship on flow angle (angle between convection and the radar line of sight) for the Doppler shift and an approximately sinusoidal relationship for the spectral width. The Doppler shift was furthermore proportional to the ion acoustic speed, ascertained from the convection speed using an established empirical formula. Taken together, these findings implied a means of estimating the convection speed and direction from coherent scatter spectra obtained at a single station. Similar spectral properties

Published by Copernicus Publications on behalf of the European Geosciences Union. 
have been reported for equatorial E-region plasma irregularities observed under counter-electrojet conditions when only Farley-Buneman waves are present and the flow angle is known (Woodman and Chau, 2002). Recent computer simulations of Farley-Buneman waves in the ionosphere also predict similar spectral behavior (Oppenheim et al., 2008). In addition, plasma convection estimates based on E-region coherent scatter spectra compare favorably with incoherent scatter measurements (Hysell et al., 2008b ${ }^{1}$ ).

In this paper, we report on a similar analysis performed with imaging radar and rocket data from the JOULE II experiment. Different conditions were encountered during JOULE I and JOULE II, affording the opportunity to test the robustness of the Bahcivan et al. (2005) results. More and better instrumentation (including the PFISR) was also available during JOULE II for the investigation. Theoretical progress in the understanding of Farley Buneman waves has also been made in the intervening years which elucidate the experimental results. The new observations support the argument that the Doppler spectra from the radio aurora embody invertible information about the magnitude and direction of the auroral electric field (Bahcivan et al., 2005). Using radar imaging, this information can be collected with relatively fine spatial and temporal resolution.

\section{Observations}

Figure 1 shows convection electric field estimates derived from PFISR incoherent scatter radar observations during the evening of 19 January 2007. The estimates are based on long-pulse F-region line-of-sight Doppler shift measurements along seven PFISR pointing positions. L-shell invariance is assumed in the estimation of vector convection profiles versus magnetic latitude at a given time (see Heinselman and Nicolls (2008) for a description of the methodology). While this assumption is required to estimate vector plasma drifts from a single station, it can be violated by auroral flows, with effects that can be difficult to assess. The fundamental time resolution of the PFISR data is about $11 \mathrm{~s}$, but for this figure, additional integration was performed to improve the statistics, yielding the plotted resolution of about 4 min. Given this integration time, the statistical uncertainty is still very significant beyond about 67.5 degrees magnetic latitude. The vector drifts shown reflect magnetic coordinates.

The convection shown in Fig. 1 was slow $(<400 \mathrm{~m} / \mathrm{s})$ except around 09:45 UT, when the convection reversed from westward to eastward, and again between about 11:3013:00 UT, when the convection was nearly eastward but had a small southward component, especially around 12:30 UT.

\footnotetext{
${ }^{1}$ Hysell, D. L., Michhue, G., Nicolls, M. J., Heinselman, C. J., and Larsen, M. F.: Assessing auroral electric field variance with coherent and incoherent scatter radar, J. Atmos. Sol. Terr. Phys., submitted, 2008.
}

The most rapid convection speed shown south of 67.5 degrees here is about $1200-1300 \mathrm{~m} / \mathrm{s}$ and occurred around the time of the rocket launches.

Figure 2 shows coherent scatter radar data for the same time interval. These data were acquired by the $30 \mathrm{MHz}$ imaging radar located at the High Latitude Monitoring Station (HLMS) site on Elmendorf Air Force Base in Anchorage operated by the Geophysical Institute. The radar observes backscatter from 5-m E-region field-aligned plasma density irregularities. The range and time resolution of the data in Fig. 2 are $2.5 \mathrm{~km}$ and $3.5 \mathrm{~s}$., respectively. Characteristics of the radar were described by Bahcivan et al. (2005). The main beam of the $30-\mathrm{MHz}$ radar is directed from Anchorage toward the Poker Flat range at a geographic azimuth of $14^{\circ}$. Radar range corresponds roughly to latitude, with the echoes shown here spanning latitudes between about $65^{\circ}-67^{\circ}$ geographic (add $\sim 0.35^{\circ}$ to convert to geomagnetic latitude). Echoes cannot be received from ranges much greater than those shown here because of the low radar elevation angles involved. The condition for field-aligned backscatter is also difficult to satisfy for E-region irregularities outside the range interval depicted in the figure. Note that the auroral echoes are range aliased and that their true range (indicated in the figure) is the apparent range plus the $415-\mathrm{km}$ radar interpulse period. That meteor echoes are absent at short ranges indicates that they are not range aliased in the main, however.

Echoes received during the convection reversal were relatively strong. These echoes were "type I" in nature, having large Doppler shifts (around $+500 \mathrm{~m} / \mathrm{s}$ here, with the positive sign signifying propagation toward the radar) and much narrower spectral widths (of the order of $50 \mathrm{~m} / \mathrm{s}$ here). The echoes observed between 11:30-13:00 UT meanwhile were weaker, with signal-to-noise ratios below $10 \mathrm{~dB}$. These echoes were "type II", with small Doppler shifts (positive and negative) and larger spectral widths (between 100$200 \mathrm{~m} / \mathrm{s}$ in this case). (See Farley, 1985a, for a definition of type I and type II spectra.) We do not associate the relative weakness of the second group of echoes with the fact that they were type II, as strong type II echoes with signal-tonoise ratios approaching $30 \mathrm{~dB}$ are observed frequently by the radar. The electron density measured by rockets in the E-region was about $2 \times 10^{5} \mathrm{~cm}^{-3}$, and so low plasma density was certainly not the cause. Pulsating aurora was visible during the second half of Fig. 2, and ground-based imagery indicated hard particle precipitation, as did the D-region density profiles measured by the PFISR. It is therefore likely that the echoes were attenuated significantly by absorption. The HAARP riometer in Gakona measured $2 \mathrm{~dB}$ absorption levels during this time. The absorption for the $30 \mathrm{MHz}$ radar should have been much more severe in view of the long slant range of the low-elevation D-region radar ray paths involved.

There is an obvious general correspondence between the flow features in Fig. 1 and the coherent echoes in Fig. 2. The irregularities observed by the $30 \mathrm{MHz}$ radar are caused by Farley Buneman instabilities and appear when 


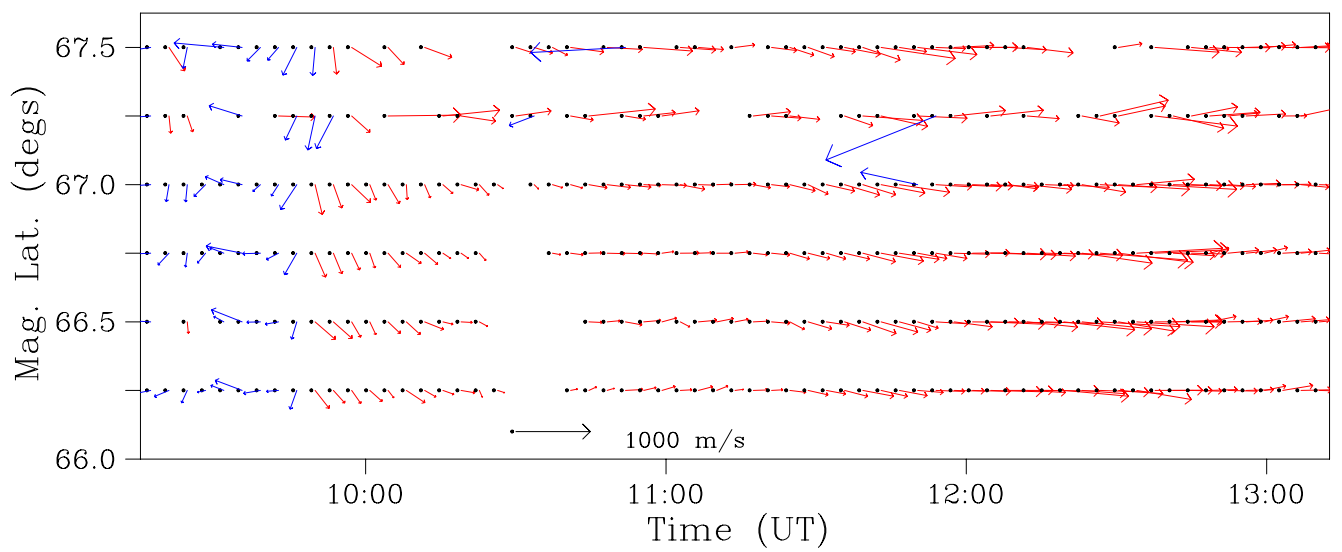

Fig. 1. Convection pattern estimated from PFISR incoherent scatter data. The horizontal axis represents UT=LT+9h. The convection reversed from westward to eastward at about 09:45 UT. Velocity estimates with relative errors less than $25 \%$ and absolute errors less than $200 \mathrm{~m} / \mathrm{s}$ are plotted. A few outliers remain, such as the spurious "reversal" just prior to 12:00 UT.

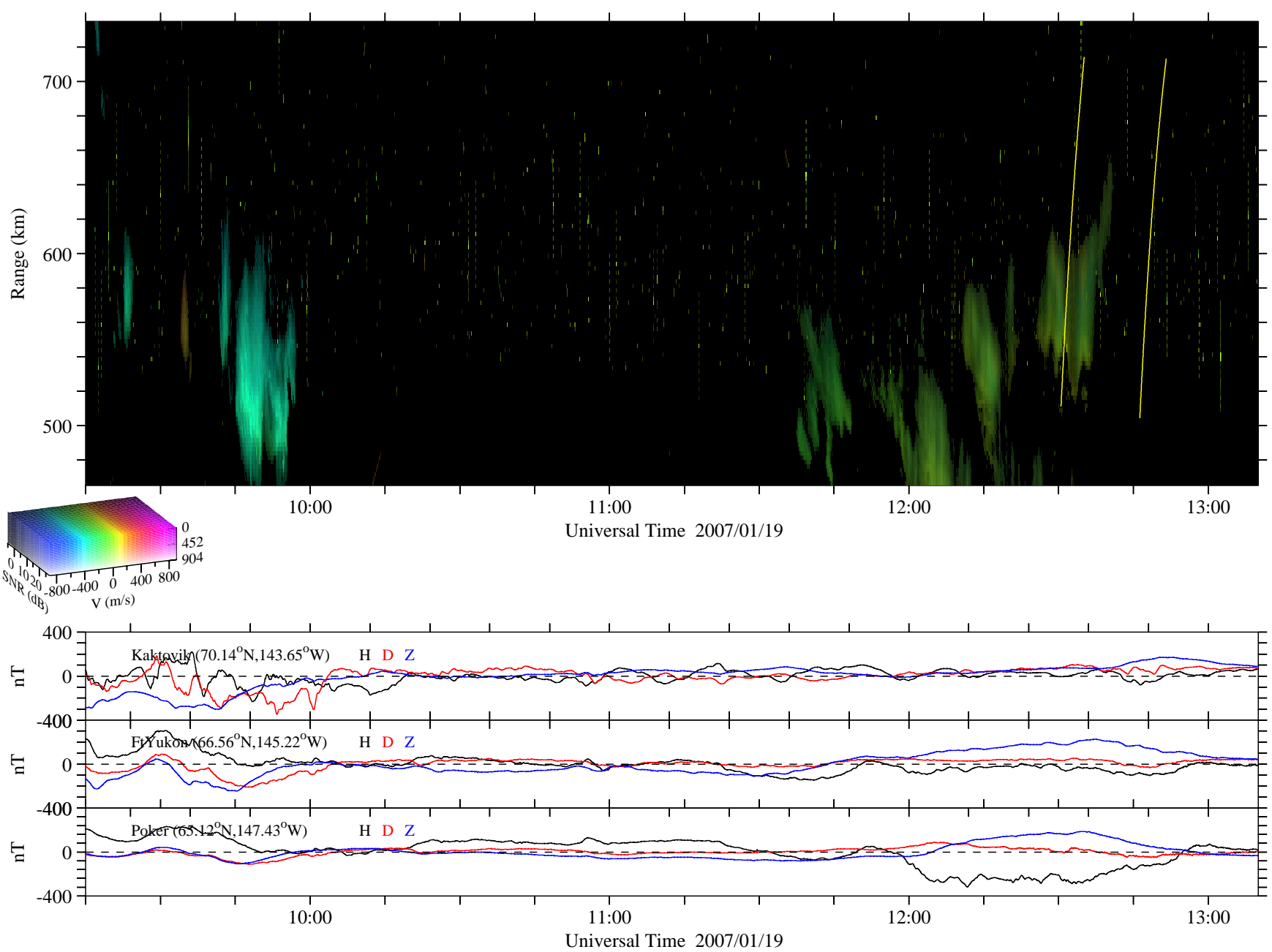

Fig. 2. Range time intensity (RTI) plot of coherent scatter received by the $30 \mathrm{MHz}$ imaging radar in Anchorage. The brightness, hue, and saturation of the pixels reflect the signal-to-noise ratio, Doppler shift, and spectral width of the echoes according to the legend shown. The solid curves are the range to the E-region footprints of the trajectories of rockets 36.234 and 21.138. Also shown are magneometer traces for Kaktovik, Ft. Yukon, and Poker Flat. 
the convection electric field (speed) exceeds about $20 \mathrm{mV} / \mathrm{m}$ (about $400 \mathrm{~m} / \mathrm{s}$ ), which is the nominal threshold for instability (e.g. Fejer et al., 1975). The Doppler shifts and spectral widths of the radar echoes are also apparently related to the magnitude and direction of the convection. Large Doppler shifts (spectral widths) accompany large line-of-sight drifts (transverse drifts). Close inspection reveals that the signs of the Doppler shifts nearly track the sign of the line-of-sight convection. Similar remarks hold generally for other combined radar observations made on other days surrounding the rocket experiments.

Instrumented rockets were launched at 12:29 UT (36.234) and 12:45 UT (21.138) following the magnetometer deflection that occurred at Poker Flat at 12:00 UT. The curves on the right side of Fig. 2 represent the range to the E-region footprints of the two rocket trajectories, mapped along magnetic field lines to an altitude of $100 \mathrm{~km}$. The first rocket flight occurred during the decaying phase of a patch of irregularities when the convection speed was still above threshold just north of the Poker Flat range. By the time the second rocket was launched, the electric field (as measured by that rocket) was below threshold everywhere in the volume visible to the $30 \mathrm{MHz}$ radar, and no backscatter was detected. The remainder of the paper therefore concentrates on data from 36.234 .

The vector electric fields measured by 36.234 are generally consistent with the convection pattern shown in Fig. 1 (see discussion surrounding Fig. 5). The convection was found to be mainly eastward with a small southward component implying line-of-sight convection toward the $30 \mathrm{MHz}$ radar. The overall morphologies of the two measurements were similar, both exhibiting extrema near the midpoint of the rocket trajectory. However, the rocket measured convection speeds as rapid as $1500 \mathrm{~m} / \mathrm{s}$ near the midpoint, above the $1200-1300 \mathrm{~m} / \mathrm{s}$ measured by the radar. It is likely that the relatively coarse resolution of the incoherent scatter data and, to some degree, the assumption of spatial homogeneity implicit in the regularization method used to construct the two-dimensional pattern in Fig. 1 are at the root of the disparity. An additional potential source of error is the imprecise mapping of small (horizontal) spatial scale electric field variations with altitude. Limitations of the ISR technique are discussed by Heinselman and Nicolls (2008).

Meaningful, quantitative comparison between rocket and 30-MHz radar data require the latter to be resolved spatially not only in range but also in azimuth. Spaced antenna techniques and statistical inverse theory are used to construct true images from the $30 \mathrm{MHz}$ coherent scatter data. Images for different Doppler spectral components are computed separately and then combined into a composite. Images are constructed in three spatial dimensions, but the elevation dimension is noninformative and is integrated out, resulting in twodimensional images in azimuth and range. The azimuth resolution for the images is about $0.4^{\circ}$. The technique was reviewed recently by Hysell and Chau (2006). Bahcivan et al.
(2006) showed recently that there is an excellent correspondence between the radar images and conventional optical auroral imagery and that features in the radar images tend to be collocated with the edges of optical auroral forms.

Figure 3 shows radar images of patches of auroral irregularities observed around 09:45 UT (top row) and 12:30 UT (bottom row) when the convection was roughly parallel to and normal to the radar line of sight, respectively. The volume probed by the radar is limited in range and azimuth by the antenna radiation pattern and the scattering geometry, but all the irregularities shown here lie well within the volume where echoes can normally be detected. The contours in Fig. 3 represent the altitudes where the condition for fieldaligned backscatter is satisfied for the radar in Anchorage, which lies off the bottom left corner of the images. That condition is nowhere satisfied for irregularities above about $97 \mathrm{~km}$. Results from the JOULE I experiment and other studies indicate that refraction, combined with finite aspect sensitivity, make it possible nevertheless to detect strong coherent backscatter from the auroral E-region between Poker flat and Arctic Village whenever the electric field is above threshold for Farley Buneman instability within the radarilluminated volume. Earlier in the day of the JOULE II experiment, auroral echoes completely filled the $30 \mathrm{MHz}$ radar range raster. Similar remarks hold for the $50 \mathrm{MHz}$ HLMS radar located nearby. This radar, which runs continuously, has a lower horizon and a broader H-plane radiation pattern than the $30 \mathrm{MHz}$ radar and is able to detect strong auroral Eregion echoes routinely over ranges between $300-1200 \mathrm{~km}$.

Each pixel in the images represents a complete Doppler spectrum. The brightness, hue, and saturation of the pixels reflect the echo intensity, Doppler shift, and spectral width according to the scales shown. Signal-to-noise ratios spanning 3-33 dB (top row) and $-3-23 \mathrm{db}$ (bottom row) are represented by the brightness. Red- and blue-shifted echoes are color coded accordingly. Phase velocities between $\pm 904 \mathrm{~m} / \mathrm{s}$ can be measured without aliasing using standard pulse-topulse analysis. Narrow and wide spectra are assigned pure and pastel tones, respectively. The incoherent integration times for these images is about $3.5 \mathrm{~s}$.

The first row of images represent a period during the convection reversal when the convection was toward the radar and above threshold. The associated spectra are narrow, with Doppler shifts around $500 \mathrm{~m} / \mathrm{s}$ and the spectral width (standard deviation) around $50 \mathrm{~m} / \mathrm{s}$. The echoes are dynamic and rapidly evolving, with sharp physical boundaries.

The second row of images represent conditions during the 36.234 rocket flight. Recall that the convection was from west to east with a small southward component. The Doppler spectra were relatively broad, with small, positive and negative Doppler shifts suggesting small flows toward and away from the radar, respectively. The images show some fine structure, especially in the aurora over Poker Flat. Dynamic behavior in the physical morphology and the spectral moments of the echoes is also evident to the north of Poker Flat. 

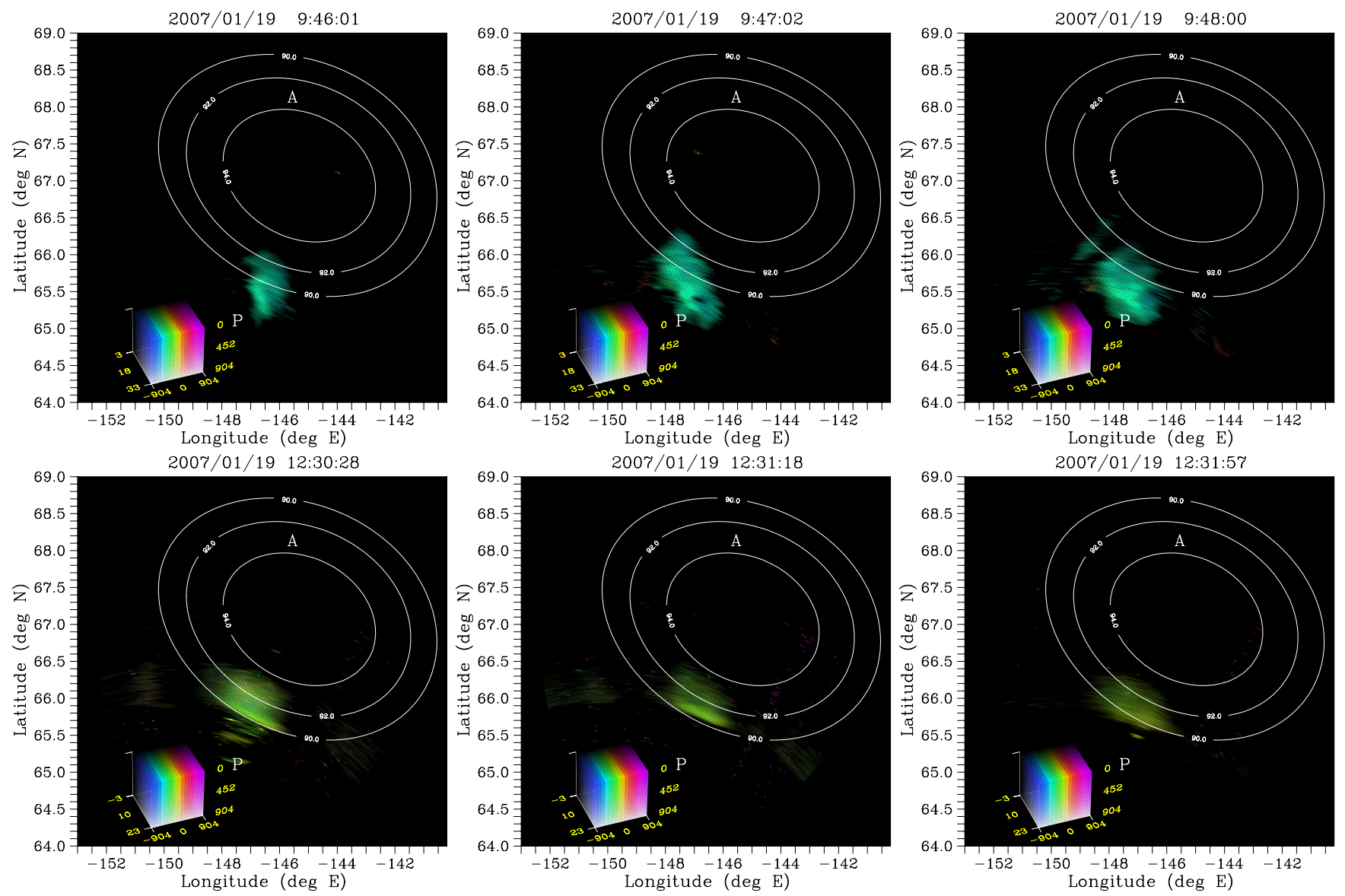

Fig. 3. Radar images from 19 January 2007. The brightness, hue, and saturation of the image pixels represent signal-to-noise ratio, Doppler shift, and spectral width on the legend shown. The integration times for the images is about $3.5 \mathrm{~s}$. The "P" and "A" symbols give the locations of the Poker Flat range and Arctic Village, respectively. Contours show the altitudes where the condition for field-aligned backscatter is satisfied from Anchorage in the absence of refraction (see text).

The echoes were relatively weak and weakened throughout the interval shown. A strong scattering ridge just inside the $90 \mathrm{~km}$ contour persisted throughout most of the interval.

Neutral winds were also measured during the JOULE II experiment. Figure 4 shows neutral wind estimates inferred from chemiluminiscent TMA trails released by another sounding rocket and photographed during the flight of 36.234. Zonal and meridional wind speed profiles are plotted separately. Also plotted is the wind speed in the direction of the main beam of the $30 \mathrm{MHz}$ radar.

The radar line-of-sight winds are very small between 105$115 \mathrm{~km}$ altitude, the nominal altitude range for auroral zone Farley Buneman waves. Similar wind profile measurements during JOULE I led Bahcivan et al. (2005) to discount any effect of neutral winds on their Farley Buneman wave observations. However, wave data from both instrumented rockets indicate that the plasma irregularities during JOULE II resided at unusually low altitudes, mainly below and near $100 \mathrm{~km}$. Figure 4 shows that there was a strong meridional neutral wind jet at this altitude. The line-of-sight wind speed in the neighborhood of $100 \mathrm{~km}$ altitude averages about $100 \mathrm{~m} / \mathrm{s}$. We may expect this wind to telegraph through to the radar Doppler spectra since Farley Buneman waves are thought to propagate near the ion acoustic speed in the ion frame of reference.

\section{Rocket/radar comparison}

The vector electric fields measured by 36.234 are compared with the imaging radar data in Fig. 5. Meaningful comparison can be made between about 80-220 s flight time (seconds after $\mathrm{T} 0=12: 29: 00 \mathrm{UT}$ ), when the rocket was in the ionosphere and not too far from the radar. The comparison is made by mapping the rocket's location along geomagnetic field lines to an E-region altitude of $100 \mathrm{~km}$. The panel in the lower left corner of Fig. 5 shows the latitude and longitude of the E-region footprint for the flight times in question. The International Geomagnetic Reference Field (IGRF) was used to make the transformation. 


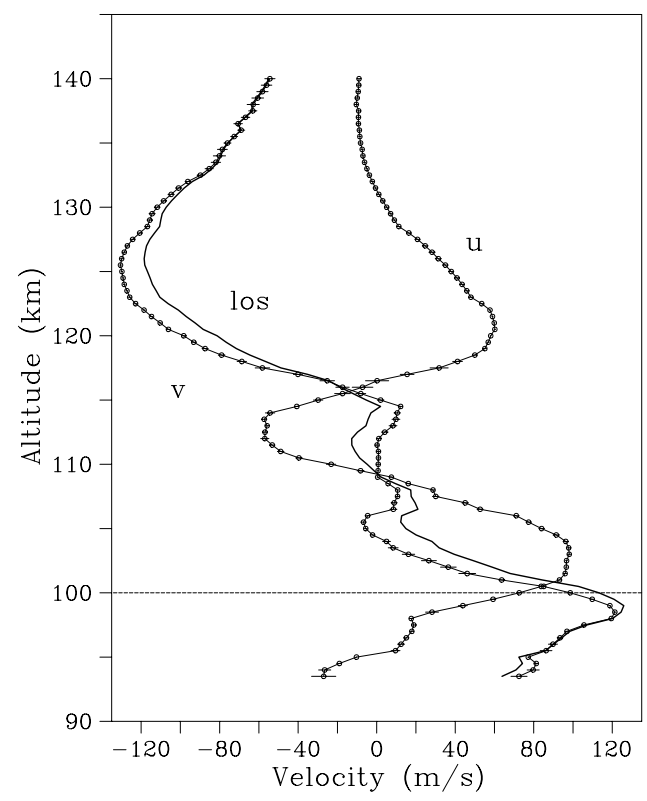

Fig. 4. Neutral winds inferred from the triangulation of TMA trails at 12:31 UT during the flight of 36.234. Curves with error bars represent meridional $(\mathrm{v})$ and zonal $(\mathrm{u})$ wind estimates, respectively. Positive values denote northward and eastward winds. The heavy curve without error bars is the wind speed in the direction of the main beam of the $30 \mathrm{MHz}$ radar (los), which is pointed toward the Poker Flat range. Positive values imply downrange winds.

IGRF was also used to convert the measured electric field to a convection velocity. The top panel of Fig. 5 shows this velocity. Here, the direction is represented by the flow angle, the angle between the convection and the radar line-of-sight to the E-region footprint. Flow angle is calculated from the vector electric field direction on the basis of the radar scattering geometry. It is measured clockwise from the radar line of sight. For flight times less than about $180 \mathrm{~s}$, the convection was mainly transverse to the line of sight but had a significant component back toward the radar. The convection speed was evidently above threshold for Farley Buneman instability almost continuously except for a brief period around $110 \mathrm{~s}$ flight time when it fell below $450 \mathrm{~m} / \mathrm{s}$.

The second panel from the top of Fig. 5 shows the range and elevation of the rocket's E-region footprint as seen from the radar, also as a function of flight time. Both the increasing range and decreasing elevation of the target imply increasing difficulty in echo detection over time. Echo detection becomes unreliable when the elevation angle falls below about $5^{\circ}$.

These electric field measurements have been reduced with a time resolution of $0.8 \mathrm{~s}$ or two points per spin. For each datum vector, we can interrogate radar data from the same time and from the the image pixel collocated with the rocket's Eregion footprint. Recall that a complete Doppler spectrum is available in each pixel. The third panel from the top of
Fig. 5 shows a spectrogram composed of spectra thus extracted from the radar data. Graycales denote the signal-tonoise ratio in each frequency bin on a scale from $-10-20 \mathrm{~dB}$. Positive Doppler frequencies denote motion toward the radar. The Doppler shifts are small and assume both signs.

The fourth panel from the top of Fig. 5 shows the moments of the radar spectrograms. The overall signal-to-noise ratio is shown as a histogram in gray. The Doppler shifts and spectral widths (standard deviations) are represented with (x) and (o) plotter symbols, respectively. These are calculated through numerical integration of the moments of the given spectra. Error bars are drawn through the plotter symbols to indicate statistical confidence. Spectral moments are only plotted when the signal-to-noise ratio exceeds a threshold of $-6 \mathrm{~dB}$.

A remarkable feature of the comparison is the close correspondence between the convection speed measured by the rocket and the echo strength measured by the radar and plotted on a logarithmic scale. The correspondence improves when the effects of the increasing range to the target is considered. Radar imagery for the entire event suggests highly variable convection in both space and time, and the agreement between rocket and radar data in this regard implies accurate spatio-temporal registration between the two measurements. Similar remarks held for the JOULE I experiment results.

The Doppler shifts track trends in the flow angle of the convection fairly closely. However, whereas the flow angle crosses $90^{\circ}$ at about $180 \mathrm{~s}$ flight time, the Doppler shift crosses zero much earlier, at about $150 \mathrm{~s}$ flight time. The two quantities appear to be vertically offset, an effect that we will attribute to the neutral wind. The Doppler width, meanwhile, appears to scale with the convection speed. Spectral broadenings occur at about $85 \mathrm{~s}$ and again at about $130 \mathrm{~s}$ flight time when the convection was strongest and the echoes were intensified. The spectra are narrowest when the convection electric field is near threshold.

In view of the results from the JOULE I experiment reported by Bahcivan et al. (2005), we can attempt to recover the spectral behavior using the same simple physical/empirical model. That model interprets radar backscatter as if arriving from hard targets, which are taken to be the corpuscular plasma density irregularities that appear in numerical simulations of the FBGD (Farley Buneman gradient drift) instability (Oppenheim et al., 1996). The irregularities move in the direction of the background convection at a uniform speed close to the presumptive ion acoustic speed $C_{s}$ in the ion frame of reference. This is the condition for marginal stability according to linear theory. According to Nielsen and Schlegel (1985); Nielsen et al. (2002), the ion acoustic speed obeys $\hat{C}_{s} \approx 400+1.1 \times 10^{-4} V_{d}^{2}$, where $V_{d}$ is the convection speed. Such a dependence on $V_{d}$ can be attributed quantitatively to wave heating (Milikh and Dimant, 2002, 2003; Bahcivan, 2007). This longitudinal irregularity 

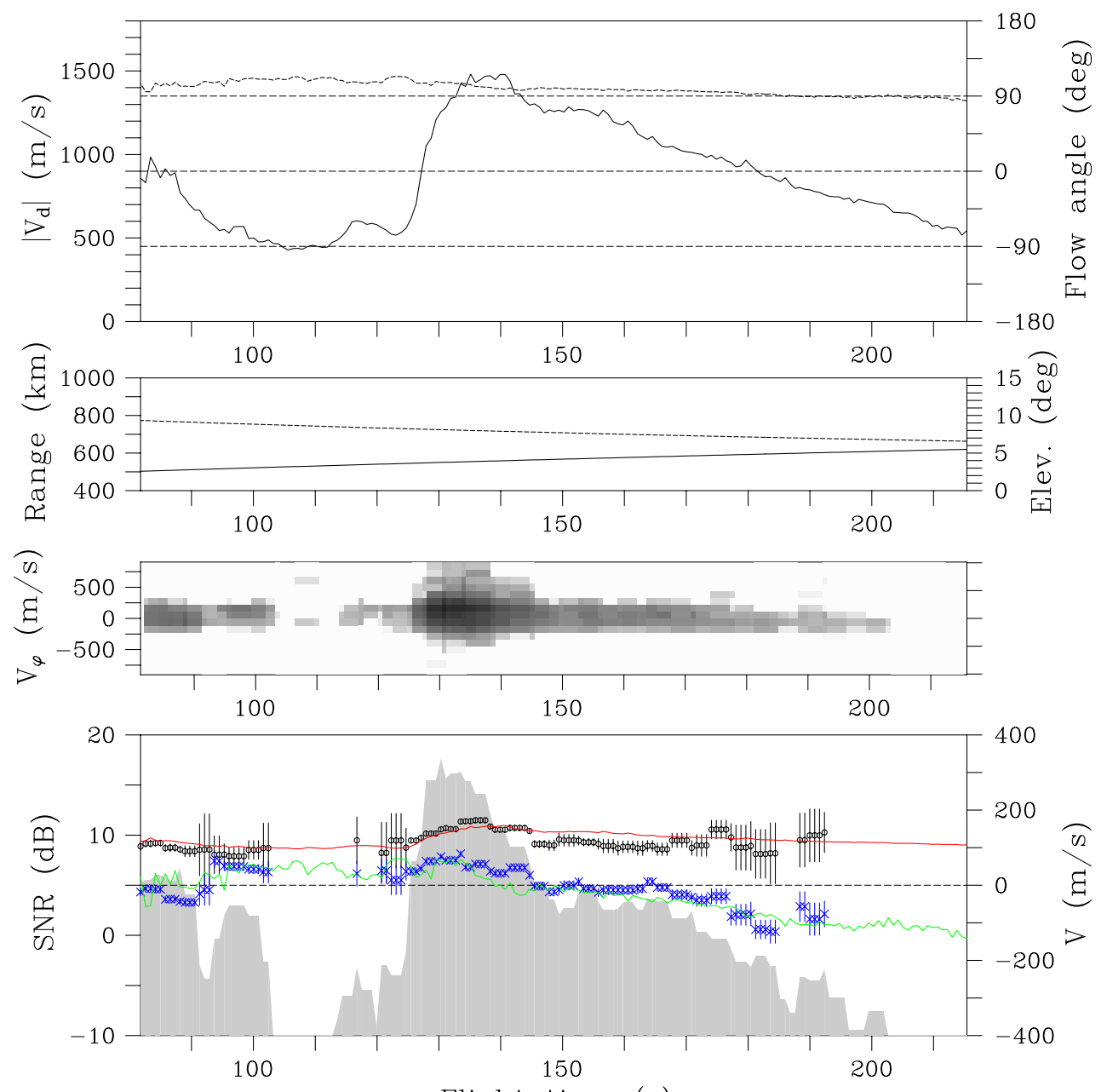

Flight time ( $s)$

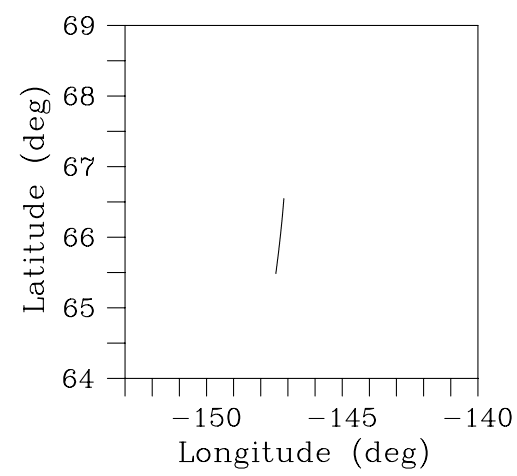

Fig. 5. Comparison of rocket and coherent scatter radar data. $T 0=12: 29: 00$ UT. First panel: rocket-derived convection speed (solid line) and direction (dashed line) as seen from the radar point of view. Second panel: range (solid line) and elevation angle (dashed line) of corresponding volume from the radar point of view. Third panel: radar spectrogram. Fourth panel: coherent scatter power (gray field), Doppler shift (x), and spectral width (o). Green and red lines represent theory (see text). Fifth panel: coordinates of the region sampled by the rocket, mapped along geomagnetic field lines to $100 \mathrm{~km}$ altitude.

motion affects the Doppler shift of the echoes to the extent it has a projection along the radar line of sight.

In the simulations, the corpuscular irregularities also move transverse to the convection in either direction at a speed that is a fraction of $C_{s}$. The sign of transverse drift speed alternates in different phases of the primary Farley Buneman wave. The symmetric transverse motion of the irregularities should not affect the mean Doppler shift according to the 
hard target model but contributes to the spectral width to the extent it has a projection along the radar line of sight. All together, the model Doppler shift (mean phase velocity) and spectral width (standard deviation) are specified in the model by:

$$
\begin{aligned}
\bar{\omega} / k & =\hat{C}_{s}\left(V_{d}\right) \cos \theta+v \\
\delta \omega_{\mathrm{rms}} / k & =\alpha \hat{C}_{s}\left(V_{d}\right)|\sin \theta|
\end{aligned}
$$

where $k$ is the radar scattering wavenumber, $\theta$ is the flow angle and $v$ is the line-of-sight neutral wind speed at the scattering altitude. Taking $v=-100 \mathrm{~m} / \mathrm{s}$ in view of Fig. 4 and tentatively setting $\alpha=1 / 4$ produces the green and red curves in Fig. 5 for Eqs. (1) and (2), respectively. The simple model curves are able to reproduce the spectral moments, given the appropriate value of the constant $\alpha$.

Data from the JOULE I experiment (vehicle 21.131) on 27 March 2003 are plotted in Fig. 6 for comparison. These data were processed in the same manner as the JOULE II data. The red and green curves in the fourth panel from the top of the figure were also generated in the same way, with two exceptions. For one, the neutral wind $v$ at the altitude of the irregularities was assumed to be zero. For another, the $\alpha$ parameter in Eq. (2) was set this time to $\alpha=1 / 2$.

The next section of the manuscript proposes an rationale for (2) and an interpretation of the $\alpha$ parameter, suggesting why it may vary from one event to another. Values for $\alpha$ close to $1 / 2$ appear to account for most of our auroral observations and also equatorial observations made during counterelectrojet conditions (Hysell et al., 2008b ${ }^{1}$; Woodman and Chau, 2002). However, the $\alpha$ scaling has not been thoroughly investigated. Numerical simulations testing the universality and sensitivity of $\alpha$ to background parameters would be revealing.

\section{Analysis}

The main findings of the Joule II imaging radar/rocket data comparison are that:

1. E-region coherent scatter was observed when the convection speed exceeded $450 \mathrm{~m} / \mathrm{s}$.

2. The convection speed and echo intensity on a logarithmic scale were highly correlated and regular.

3. The Doppler shifts of the radar echoes tracked the product of the cosine of the flow angle and the presumptive ion acoustic speed calculated according to an empirical formula, $\hat{C}_{s}$. The Doppler shifts were offset from the formula $w / k=\hat{C}_{s} \cos \theta$ by $100 \mathrm{~m} / \mathrm{s}$. This matched the line of sight neutral wind speed at the observed irregularity altitude of $100 \mathrm{~km}$. Unmeasured horizontal and temporal variations in the line-of-sight wind most likely contributed to the discrepancy between the Doppler shift measurements and the model curves.
4. The spectral width was proportional to the same presumptive ion acoustic speed times the sine of the flow angle. The proportionality constant was precisely $1 / 4$ throughout the event. The spectrum becomes narrow at small flow angles, presumably approaching some yet undetermined minimum width.

The results are reminiscent with those obtained during the JOULE I experiment and presented by Bahcivan et al. (2005), with a few discrepancies. The JOULE I echoes were much stronger than the JOULE II echoes, which likely suffered from enhanced ionospheric absorption. The JOULE I event was also characterized by much larger flow angle excursions, allowing a more comprehensive assessment of flow angle effects than is possible here. In JOULE I, the coherent scatter spectra were observed to transition from broad to narrow as the flow angle moved closer to parallel to the radar line of sight. Only broad spectra were observed during JOULE II. Furthermore, the irregularities resided at higher altitudes in the JOULE I events than in JOULE II. The threshold for irregularity excitation was only $400 \mathrm{~m} / \mathrm{s}$ in JOULE I, versus $450 \mathrm{~m} / \mathrm{s}$ in JOULE II. According to linear theory, Farley Buneman waves should be excited when the convection speed exceeds the product of the ion acoustic speed times the factor $1+\psi$, where $\psi$ is the anisotropy factor (e.g. Fejer et al., 1995). At $100 \mathrm{~km}$ altitude, $\psi$ is approximately 0.15 which is large enough to account for the higher JOULE II instability threshold.

The JOULE II irregularities also resided in a stratum with a $100 \mathrm{~m} / \mathrm{s}$ line of sight neutral wind. This appears to have resulted in a corresponding offset to the echo Doppler shifts, which changed sign at different times than the projection of the convection velocity on the radar line of sight. This is also consistent with linear theory, which states that the condition for marginal stability of Farley Buneman waves is that the phase speed equal the ion acoustic speed in the ion frame of reference, which is the same as the neutral frame of reference at $100 \mathrm{~km}$ altitude.

A recent numerical study of the FBGD instability performed by Oppenheim et al. (2008) provides a theoretical basis for the observations reported here. Their study involved the highest resolution two-dimensional particle-in-cell simulation of the nonlinear, fully kinetic instability performed to date. The simulations produced primary Farley Buneman waves which propagated with a phase speed less than the convection speed and close to the ion acoustic speed. Ascertaining the actual value of the ion acoustic speed is complicated because the ratios of the specific heats of the electrons and ions are not well established, but the authors found no contradiction between their simulation results and the assertion that primary Farley Buneman waves propagate at something close to the ion acoustic speed in nature.

The Oppenheim et al. (2008) simulations also showed secondary waves propagating obliquely to the primary waves. The secondaries are driven by convection due to 

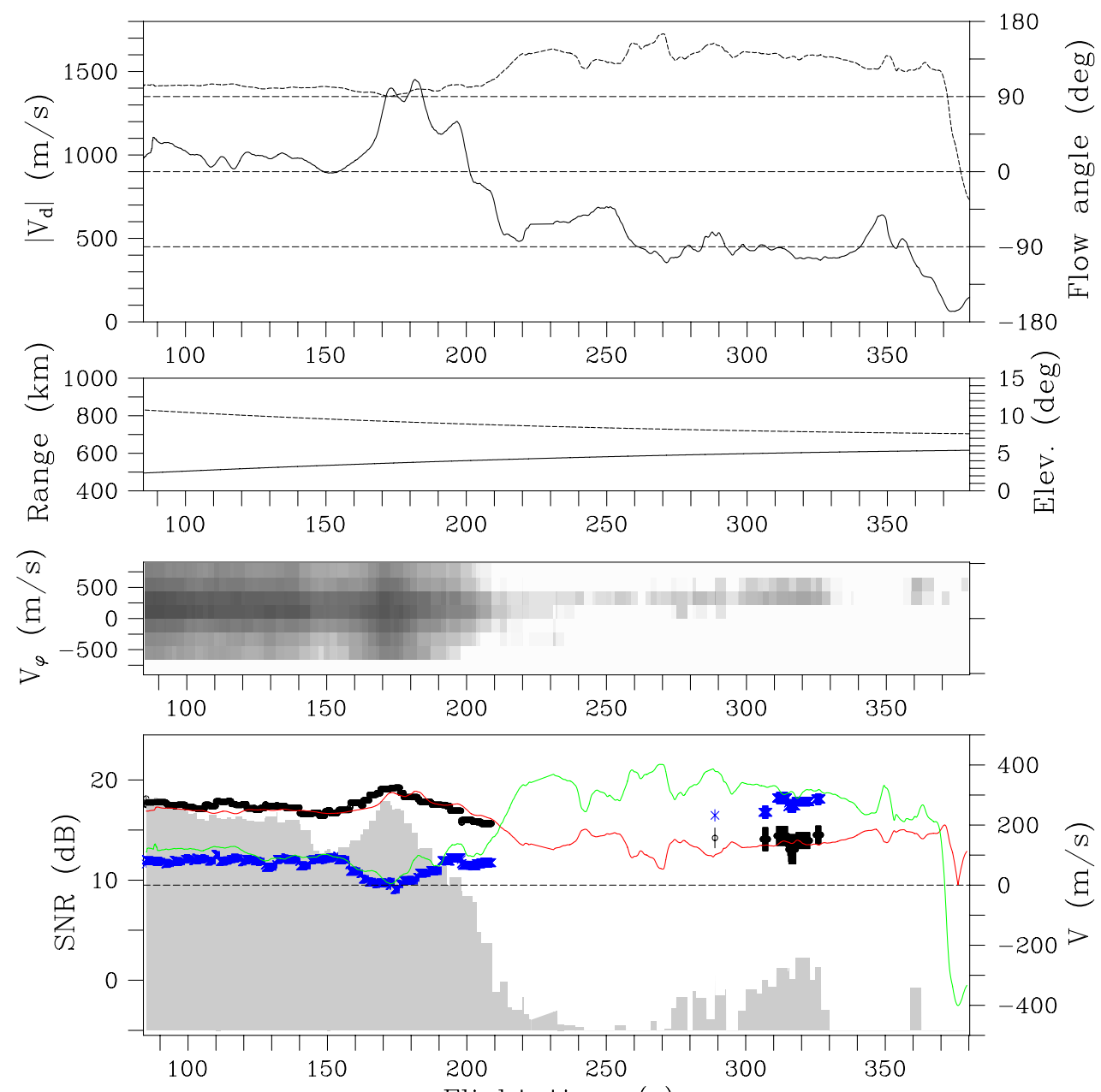

Flight time (s)

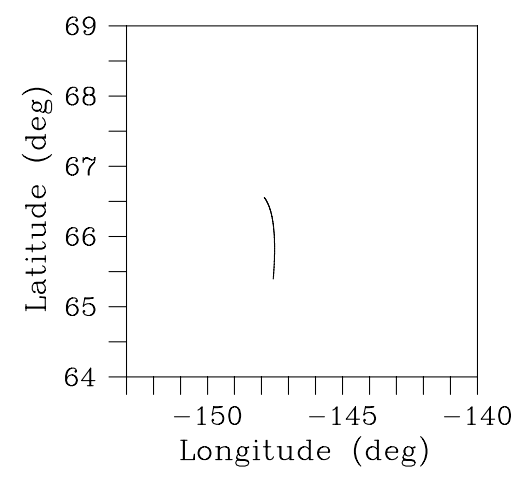

Fig. 6. Same as previous figure, only for JOULE I rocket flight 21.131 on 27 March 2003. Here, T0=12:12:01 UT. Data were processed in the same manner as the JOULE II data.

the polarization electric field of the primary and are also affected by the density gradient associated with the primary. The superposition of the primary and secondaries produced what appeared to be trains of corpuscular density irregularities moving in bulk in nearly in the direction of the background convection. (Similar morphology is seen in the model studies of Otani and Oppenheim, 2006, which considered nonlinear mode coupling between just three waves.) Oppenheim et al. (2008) calculated spectrograms of all the waves in their simulation satisfying the Bragg scattering condition for different scattering wavelengths as a function of flow angle. Their results showed an unmistakable cosine dependence of Doppler shift on flow angle for the scattering wavelengths between 0.75 and $6 \mathrm{~m}$ they considered. At zero flow angle, 


\section{primary wave trough}

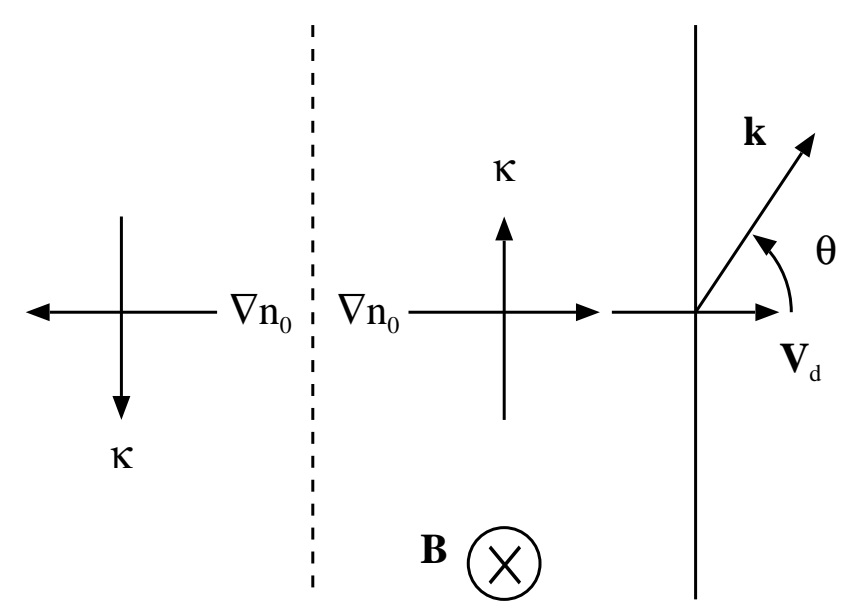

Fig. 7. Diagram illustrating the spectral width model developed in Sect. 4.

the spectrograms were narrow with phase velocities close to $C_{s}$. At other flow angles, the spectra were broader. Overall, the widths of the spectrograms exhibited a sinusoidal dependence on flow angle, with maximum widths a fraction of $C_{s}$ associated with propagation normal to the convection. The spectrograms showed comparable wave energies at all flow angles for $6 \mathrm{~m}$ irregularities. At shorter wavelengths, the wave energy became more anisotropic and concentrated at small angles. This is consistent with fact that type I (type II) echoes dominate backscatter at frequencies above (below) about $50 \mathrm{MHz}$ (Balsley and Farley, 1971; Meyer et al., 2004).

Oppenheim et al. (2008) interprets the saturation of the phase speed of the primary Farley Buneman wave at something close to the ion acoustic speed as a consequence of mode coupling to secondary, linearly stable FBGD waves propagating obliquely to the primaries. Mode coupling maintains a statistical steady state whereby the primary waves are operating near the linear threshold for marginal stability. Although their simulations are two dimensional, similar behavior is predicted by the semi-heuristic model of Milikh and Dimant (2002) which includes coupling to off-perpendicular modes. Off-perpendicular wave modes contribute substantially to wave heating, causing the electron temperature and the ion acoustic speed to increase in a manner consistent with the empirical formula of Nielsen and Schlegel (1985). The usual formulation for the primary wave eigenfrequency may be made to agree with this picture through the inclusion of appropriate anomalous and finite parallel wavenumber effects, although this is not very clear.

A more powerful and intuitive interpretation of the phase speeds of Farley Buneman waves was offered by J. P. St. Maurice (personal communication) and then Drexler (2005) who noted a peculiar quality of the waves. Accord- ing to the linear dispersion relation, the phase velocity of any wave (primary or secondary, with any aspect angle) is the projection of the group velocity of the wave packet in the direction of the wavevector, i.e. $\omega=\mathbf{k} \cdot \mathbf{v}_{g}$. In view of the results of Oppenheim et al. (2008), the group velocity must have magnitude and direction close to the ion acoustic speed and the convection direction, respectively. This interpretation therefore supports the $\bar{\omega} / k=C_{s} \cos \theta$ model. Since the phase speed saturation refers to the ion frame of reference, line-of-sight neutral wind-induced ion motion will appear as an additive Doppler shift.

We can use the Doppler shift model to construct a spectral width model by assuming that primary and secondary waves alike obey the condition for marginal linear stability and that marginal stability is maintained even though the system is forced well beyond the threshold for instability. We further presume that the primary wave has a much longer wavelength than the secondary waves for simplicity, although this is consistent neither with observations nor simulations. Standard linear, local, two-fluid analysis produces the following equation governing the behavior of the Fourier density mode $n_{\mathbf{k}}(t)$. We understand this mode to be the secondary mode satisfying the Bragg scattering condition for the radar. Different frequency components of the modal amplitude correspond to different frequency bins of the Doppler spectrum. Note that the analysis here shares similarities with the one by Hamza and St-Maurice (1993a), although the interpretation is different. Our analysis is purely linear and does not explicitly associate spectral broadening with nonlinear terms in the system.

$$
\begin{aligned}
& \left(\frac{\partial}{\partial t}+i \frac{\mathbf{k} \cdot \mathbf{V}_{d}}{1+\psi}\right) n_{\mathbf{k}} \\
& =-\frac{1}{1+\psi}\left\{\frac{\psi}{v_{i}}\left(\frac{\partial^{2}}{\partial t^{2}}+k^{2} C_{s}^{2}\right)+i \frac{\mathbf{k} \cdot \kappa}{k^{2}} \frac{v_{i}}{\Omega_{i}} \frac{\partial}{\partial t}\right\} n_{\mathbf{k}} \\
& \kappa \equiv \frac{\nabla n_{\circ}}{n_{\circ}} \times \hat{b}
\end{aligned}
$$

where $\mathbf{V}_{d}$ is the background convection velocity, $n_{\circ}$ is the background plasma density, $\psi$ is the anisotropy factor, and where the other terms have their familiar meaning. Refer to Fig. 7 for an illustration of the model geometry. In writing this equation for the secondary waves, we assume the presence of a primary wave and include the associated electric field and density variations in $\mathbf{V}_{d}$ and $n_{\circ}$, respectively. The $\kappa$ term therefore arises from the primary wave in an ionosphere otherwise taken to be homogeneous.

Multiplying both sides of Eq. (3) by $n_{\mathbf{k}}^{*}$ converts the equation to one governing the energy in a Fourier mode $\left|n_{\mathbf{k}}\right|^{2}$. Retaining only the real part of the equation yields:

$$
\begin{aligned}
& \frac{\partial}{\partial t}\left|n_{\mathbf{k}}\right|^{2} \\
& =-\frac{2}{1+\psi}\left\{\frac{\psi}{v_{i}} \Re\left(n_{\mathbf{k}}^{*} \frac{\partial^{2}}{\partial t^{2}} n_{\mathbf{k}}+k^{2} C_{s}^{2}\left|n_{\mathbf{k}}\right|^{2}\right)\right.
\end{aligned}
$$




$$
\left.-\Im\left(\frac{\mathbf{k} \cdot \kappa}{k^{2}} \frac{\nu_{i}}{\Omega_{i}} n_{\mathbf{k}}^{*} \frac{\partial}{\partial t} n_{\mathbf{k}}\right)\right\}
$$

For marginal linear stability, both sides of Eq. (4) should be close to zero on average, and this condition is asserted in the equations that follow.

Let us decompose $n_{\mathbf{k}}(t)$ into its frequency components according to

$n_{\mathbf{k}}(t)=\sum_{\omega} n_{\mathbf{k}, \omega} e^{-i \omega t}$

with $\omega$ then corresponding to the Doppler frequency components of backscatter associated with a scattering wavevector k. The marginal stability condition then becomes:

$$
\left\{\frac{\psi}{v_{i}}\left(\omega^{2}-k^{2} C_{s}^{2}\right)+\frac{\mathbf{k} \cdot \kappa}{k^{2}} \frac{v_{i}}{\Omega_{i}} \omega\right\}\left|n_{\mathbf{k}, \omega}\right|^{2}=0
$$

The $\omega^{2}$ term is related to Farley Buneman wave growth associated with the action of ion inertia, and the second term to dissipation due to ambipolar diffusion. The remaining term is associated with growth due to the gradient drift instability. This term is generally thought to be able to modify the threshold condition for the onset of Farley Buneman instability depending on the magnitude and direction of the background density gradient (Farley and Fejer, 1975). Recall that in the present context, we associate $\nabla n$ 。 with plasma density variation of the primary wave. The term can therefore be quite large. This should have the effect of reducing the threshold condition for secondary wave growth and, therefore, of reducing the phase speeds of the secondary waves below $C_{s}$. The primary wave also drives the transverse convection that excites the secondaries, an assumption implicit in this analysis.

The final step in this analysis is to sum Eq. (5) over all frequencies. Define the following spectral moments:

$$
\begin{aligned}
\bar{\omega} & =\sum_{\omega} \omega\left|n_{\mathbf{k}, \omega}\right|^{2} / \sum_{\omega}\left|n_{\mathbf{k}, \omega}\right|^{2} \\
\overline{\omega^{2}} & =\sum_{\omega} \omega^{2}\left|n_{\mathbf{k}, \omega}\right|^{2} / \sum_{\omega}\left|n_{\mathbf{k}, \omega}\right|^{2} \\
& =\overline{(\omega-\bar{\omega})^{2}}+\bar{\omega}^{2} \\
& =\overline{\delta \omega^{2}}+\bar{\omega}^{2}
\end{aligned}
$$

Neglecting the gradient drift term for the moment, Eq. (5) then becomes (after summing over all frequencies):

$\overline{\delta \omega^{2}}+\bar{\omega}^{2}=k^{2} C_{s}^{2}$

which is to say that the sum of the squares of the spectral width and the Doppler shift should equal to $C_{s}^{2}$. While this is consistent with the assertion of sine and cosine dependencies on the flow angle for the two, respectively, it overestimates the spectral width considerably due to the neglect of the gradient drift term. This term requires careful treatment in view of the fact that $\kappa$ and $\omega$ are correlated in the present context in some sense.
Since the background density gradients associated with the primary wave are longitudinal, we may write $\mathbf{k} \cdot \kappa=k \kappa \sin \theta$. Next, we define

$\overline{\kappa \omega}=\sum_{\omega} \kappa \omega\left|n_{\mathbf{k}, \omega}\right|^{2} / \sum_{\omega}\left|n_{\mathbf{k}, \omega}\right|^{2}$

We should expect $\left|n_{\mathbf{k}, \omega}\right|^{2}$ to be largest for those frequency components for which $\kappa \omega$ is positive, since the growth of those wave components is assisted by the gradient drift mechanism and the threshold for instability consequently suppressed. The sign of $\kappa$ is different in different phases of the primary wave, and so secondary wave components with different signed frequencies will be more or less prevalent in different phases. The two quantities are thus correlated. While we cannot calculate $\overline{\kappa \omega}$ on the basis of the present analysis, we can express it in terms of a normalized correlation coefficient:

$\mathcal{C} \equiv \frac{\overline{\kappa \omega}}{\kappa_{r m s} \delta \omega_{r m s}}$

Rewriting Eq. (5) accordingly and summing over all frequencies leads to:

$\overline{\delta \omega^{2}}+\bar{\omega}^{2}+\frac{\nu_{i} \Omega_{e}}{\nu_{e}} \frac{\mathcal{C}}{k L_{\mathrm{rms}}} \sin \theta{\overline{\delta \omega^{2}}}^{1 / 2}=k^{2} C_{s}^{2}$

with $L_{\mathrm{rms}} \equiv 1 / \kappa_{\mathrm{rms}}$ being the rms gradient lengths scale of longitudinal density irregularity associated with the primary wave. Finally, taking $\bar{\omega}=k C_{s} \cos \theta$ in accordance with the discussion above leads to an expression for the spectral width (standard deviation) that is proportional to the sine of the flow angle.

$$
\begin{aligned}
\delta \omega_{r m s} & =|\sin \theta|\left(\sqrt{k^{2} C_{s}^{2}+\Gamma^{2}}-\Gamma\right) \\
\Gamma & \equiv \frac{v_{i} \Omega_{e}}{2 v_{e}} \frac{\mathcal{C}}{k L_{r m s}}
\end{aligned}
$$

where $\Gamma$ is related to the gradient drift instability growth rate as telegraphed through to the Farley Buneman instability threshold condition. The frequency ratio in $\Gamma$ just reflects the relative growth rates of the two instabilities. The $k L$ term in the denominator reflects the preference of the gradient drift instability for short gradient length scales and long wavelengths. The correlation coefficient $\mathcal{C}$ reflects the fact that the irregularities will only benefit from the gradient drift mechanism to the extent they are preferentially localized in the phases of the primary wave where the growth rate is positive. As the correlation coefficient and the correction term $\Gamma$ increase together, the threshold speed for the secondary waves declines, and the spectral width estimator falls further below $k C_{s}$. We can set $k L_{\mathrm{rms}}=20$ for primary waves with $5 \%$ relative amplitude and secondary waves with wavelengths comparable to the primaries. A correlation coefficient $\mathcal{C}$ of $\approx 0.2$ then yields spectral width predictions comparable to what was observed during Joule II. 


\section{Summary}

Using radar imaging, we have compared in situ vector electric field measurements with coherent scatter Doppler spectra in the auroral E-region with very fine spatial (a few $\mathrm{km}$ ) and temporal (a few seconds) resolution. The comparison shows that the Doppler shift and spectral width of the echoes appear to be related to the cosine and sine (or perhaps other closely related monotonic functions) of the flow angle with proportionality constants related the ion acoustic speed, as estimated with an established empirical formula. The neutral wind also appears to influence the Doppler shift additively, although it is difficult to measure this parameter comprehensively. In future experiments, we plan to actually measure the electron and ion temperatures and the neutral winds in the E-region with the PFISR to fill in that part of the causality chain. The results from JOULE II are consistent with findings from the JOULE I experiment as well as from the equatorial electrojet during counterelectrojet conditions when there are no large-scale gradient waves present to complicate things (Woodman and Chau, 2002). The results are also in qualitative agreement with recent numerical simulations which reproduce the sine and cosine laws and also the limiting role of the ion acoustic speed (Oppenheim et al., 2008).

Analysis based on linear, quasilinear, weak turbulence, and strong turbulence models has had mixed success in predicting the properties of the radio aurora (see reviews by Farley, 1985b; Hamza and St-Maurice, 1993a,b). One of the more successful findings continues to be that primary Farley Buneman waves maintain a state of marginal linear stability. Not only does this account for the fact that they propagate with phase speeds near the ion acoustic speed, it also predicts their magnetic aspect width to the degree required to account for the wave heating observed (Milikh and Dimant, 2002). Once the primary waves achieve marginal linear stability, they immediately generate a spectrum of secondary waves that 1) extract energy from the primaries, limiting their amplitude, 2) heat the ionosphere (particularly secondaries with finite parallel wavenumbers), increasing the threshold speed, and 3) disrupt the convection around the primary waves, limiting their phase speeds in the manner depicted by Otani and Oppenheim (2006).

The superposition of the primary and secondary waves leads to corpuscular plasma density irregularities moving in bulk with the convection near the ion acoustic speed. These irregularities, which are seen clearly in simulations (Oppenheim et al., 1996), give rise to coherent scatter. St. Maurice (personal communication) has accounted for the cosine dependence on flow angle by pointing out that the phase speed of a scattering mode is equal to the projection of the group velocity in the direction of the scattering wavevector. This is equivalent to a hard-target interpretation of the backscatter.

In this paper, we have extended the marginal linear stability surmise to the secondary waves detected by radars prob- ing the radio aurora at finite flow angles. Linear analysis suggests that spectral width of the backscatter, which is a consequence of the transverse motion of the corpuscular irregularities under the control of the electric field of the primary wave, should exhibit a sinusoidal dependence on flow angle. The magnitude of the spectral width can fall well below the ion acoustic speed due to the destabilizing action of the gradient drift mechanism.

Radar imaging, which is a superresolution method, is an essential component of this analysis. The model in Eqs. (1) and (2) that relates the background convection to the spectral moments is nonlinear, and superposition does not hold. Fine spatial and temporal resolution is therefore essential for recovering the behavior expressed by the model. The spatiotemporal resolution of the coherent scatter radar data presented here is probably marginal in view of the degree of variability we observe in the echoes from one range gate, azimuth bin, and time stamp to the next.

Our analysis has neglected electron and ion thermal effects thought to be important in the FBGD instability under strong forcing (e.g. Dimant and Oppenheim, 2004; Oppenheim and Dimant, 2004; Kagan and St.-Maurice, 2004.) Among other possible effects is the turning of the primary wave from the electron convection direction by as much as about 30 degrees. To first order, this would imply an offset from the flow angle in our empirical formulas. Neither this study nor the one reported by Hysell et al. (2008b) ${ }^{1}$ found an obvious offset, although it is possible that an offset lies behind the discrepancies in the radar and rocket measurements and the variation in the $\alpha$ parameter. Future experiments will be conducted to address this issue.

Reasonable rocket/radar data agreement was achieved by setting the $\alpha$ parameter in Eq. (2) to a constant value. The parameter must not be a universal constant since the spectral widths were narrower during JOULE II than they were during JOULE I despite the fact that the range of convection speeds was comparable. (The spectral widths observed during the JOULE I rocket missions were greater than $300 \mathrm{~m} / \mathrm{s}$ at times.) However, the persistent rocket/radar data correspondence in Figs. 5 and 6 argues that $\alpha$ remained relatively constant throughout each mission. Equation (6) is not terribly predictive, since we cannot estimate $\mathcal{C}$ or $L_{\mathrm{rms}}$ a priori. However, the ion to electron collision frequency ratio is expected to increase somewhat with decreasing altitude, offering a possible explanation for the narrow spectra observed during JOULE II. Additional experiments and simulations can show if there is a systematic relationship between spectral width and irregularity altitude or some other measurable parameter.

Acknowledgements. D. L. Hysell is grateful for the assistance of John Peters at the HLMS station operated by the Geophysical Institute of the University of Alaska, Fairbanks. Insights offered by D. T. Farley at Cornell and M. M. Oppenheim at Boston University were greatly appreciated. The research was supported by award no. ATM-0531683 from the National Science Foundation and 
no. NNG05WC42G from the National Aeronautics and Space Administration to Cornell University and by award no. ATM-0541593 from the National Science Foundation and no. NNG05WC40G from the National Aeronautics and Space Administration to Clemson University. The PFISR was developed under NSF cooperative agreement ATM-0121483, and the data collection and analysis was supported under NSF cooperative agreement ATM-0608577.

Topical Editor M. Pinnock thanks M. Oppenheim and another anonymous referee for their help in evaluating this paper.

\section{References}

Bahcivan, H.: Plasma wave heating during extreme electric fields in the high-latitude $E$ region, Geophys. Res. Lett., 34, L15106, doi:10.1029/2006GL029236, 2007.

Bahcivan, H., Hysell, D. L., Larsen, M. F., and Pfaff, R. F.: 30 $\mathrm{MHz}$ imaging radar observations of auroral irregularities during the JOULE campaign, J. Geophys. Res., 110, A05307, doi:10.1029/2004JA010975, 2005.

Bahcivan, H., Hysell, D. L., Lummerzheim, D., Larsen, M. F., and Pfaff, R. F.: Observations of collocated optical and radar auroras, J. Geophys. Res., 111, A12308, doi:10.1029/2006JA011923, 2006.

Balsley, B. B. and Farley, D. T.: Radar studies of the equatorial electrojet at three frequencies, J. Geophys. Res., 76, 8341-8351, 1971.

Codrescu, M. V., Fuller-Rowell, T., and Foster, J.: On the importance of E-field variability for Joule heating in the high-latitude thermosphere, Geophys. Res. Lett., 22, 2393-2396, 1995.

Dimant, Y. S. and Oppenheim, M. M.: Ion thermal effects on Eregion instabilities: linear theory, J. Atmos. Sol. Terr. Phys., 66, 1639-1654, 2004.

Drexler, J.: Phase vs. group velocities in $E$ region radar observations, CEDAR Workshop plenary presentation, Santa Fe, 27-31 June 2005

Farley, D. T.: On-line data processing for mst radars, Radio Sci., 20, 1177-1184, 1985a.

Farley, D. T.: Theory of equatorial electrojet plasma waves: New developments and current status, J. Atmos. Terr. Phys., 47, 729$744,1985 b$

Farley, D. T. and Fejer, B. G.: The effect of the gradient drift term on the type 1 electrojet irregularities, J. Geophys. Res., 80, 3087, 1975.

Fejer, B. G., Farley, D. T., Balsley, B. B., and Woodman, R. F.: Vertical structure of the VHF backscattering region in the equatorial electrojet and the gradient drift instability, J. Geophys. Res., 80, 1313-1323, 1975.

Fejer, B. G., dePaula, E. R., Heelis, R. A., and Hanson, W. B.: Global equatorial ionospheric vertical plasma drifts measured by the AE-E satellite, J. Geophys. Res., 100, 5769-5776, 1995.

Foster, J. C. and Erickson, P. J.: Simultaneous observations of $E$ region coherent backscatter and electric field amplitude at $F$ region heights with the Millstone Hill UHF radar, Geophys. Res. Lett., 27, 3177-3180, 2000.

Haldoupis, C.: A review on radio studies of auroral E-region ionospheric irregularities, Ann. Geophys., 7, 239-258, 1989, http://www.ann-geophys.net/7/239/1989/.

Hamza, A. M. and St-Maurice, J. P.: A turbulent theoretical framework for the study of current-driven E region irregularities at high latitudes: Basic derivations and application to gradient-free situations, J. Geophys. Res., 98, 11 587-11 599, 1993a.

Hamza, A. M. and St-Maurice, J. P.: A self consistent fully turbulent theory of auroral $E$ region irregularities, J. Geophys. Res., 98, $11601-11613,1993 b$

Heinselman, C. J. and Nicolls, M. J.: A Bayesian approach to electric field and $E$-region neutral wind estimation with the Poker Flat Advanced Modular Incoherent Scatter Radar, Radio Sci., in press, 2008.

Hysell, D. L. and Chau, J. L.: Optimal aperture synthesis radar imaging, Radio Sci., 41, RS2003, doi:10.1029/2005RS003383, 2006.

Kagan, L. M. and St.-Maurice, J. P.: Impact of electron thermal effects on Farley-Buneman waves at arbitrary aspect angles, J. Geophys. Res., 109, A12302, doi:10.1029/2004JA010444, 2004.

Makarevich, R. A., Senior, A., Koustov, A. V., Uspensky, M., Honary, F., and Dyson, P. L.: A study of aspect angle effects in the $E$-region irregularity velocity using multi-point electric field measurements, Geophys. Res. Lett., 33, L21102, doi:10.1029/2006GL027740, 2006.

Meyer, M. G., Sahr, J. D., and Morabito, A.: A statistical study of subauroral $E$-region coherent backscatter observed near $100 \mathrm{MHz}$ with passive radar, J. Geophys. Res., 109, A07308, doi:10.1029/2004JA010396, 2004

Milikh, G. M. and Dimant, Y. S.: Kinetic model of electron heating by turbulent electric field in the $E$ region, Geophys. Res. Lett., 29(12), 1575-1578, 2002.

Milikh, G. M. and Dimant, Y. S.: Model of anomalous electron heating in the $E$ region: Detailed numerical modeling, J. Geophys. Res., 108, 1350, doi:10.1029/2002JA009524, 2003.

Moorcroft, D. R.: Outstanding issues in the theory of radar aurora: Evidence from the frequency dependence of spectral characteristics, J. Geophys. Res., 107, doi:10.1029/2001JA009218, 2002.

Nielsen, E. and Schlegel, K.: Coherent radar Doppler measurements and their relationship to the ionospheric electron drift velocity, J. Geophys. Res., 90, 3498-3504, 1985.

Nielsen, E., del Pazo, F., and Williams, P. J. S.: VHF coherent radar signals from the $E$ region ionosphere and the relationship to electron drift velocity and ion-acoustic velocity, J. Geophys. Res., 107, 1012, doi:10.1029/2001JA900111, 2002.

Oppenheim, M., Otani, N., and Ronchi, C.: Saturation of the FarleyBuneman instability via nonlinear electron $\mathbf{E} \times \mathbf{B}$ drifts, J. Geophys. Res., 101, 17 273-17 286, 1996.

Oppenheim, M. M. and Dimant, Y. S.: Ion thermal effects on Eregion instabilities: 2-D kinetic simulations, J. Atmos. Sol. Terr. Phys., 66, 1655-1668, 2004

Oppenheim, M. M., Dimant, Y., and Dyrud, L.: Large scale sumulations of 2-D fully kinetic Farley Buneman turbulence, Ann. Geophys., 26, 543-553, 2008, http://www.ann-geophys.net/26/543/2008/.

Otani, N. F. and Oppenheim, M.: Saturation of the Farley-Buneman instability via three-mode coupling, J. Geophys. Res., 111, A03022, doi:10.1029/2005JA011215, 2006

Robinson, R. T.: Towards a self consistent nonlinear theory of radar auroral backscatter, J. Atmos. Terr. Phys., 48, 417-422, 1986.

Sahr, J. D. and Fejer, B. G.: Auroral electrojet plasma irregularity theory and experiment: A critical review of present understanding and future directions, J. Geophys. Res., 101, 26 893-26 909, 1996. 
St.-Maurice, J. P., Schlegel, K., and Banks, P. M.: Anomalous heating of the polar $E$ region by unstable plasma waves, 2, theory, J. Geophys. Res., 86, 1453-1462, 1981.

Uspensky, M., Koustov, A., Janhunen, P., Pellinen, R., Danskin, D., and Nozawa, S.: STARE velocities: The importance of offorthogonality and ion motions, Ann. Geophys., 21, 729-743, 2003,

http://www.ann-geophys.net/21/729/2003/.
Uspensky, M., Koustov, A., Janhunen, P., Nielsen, E., Kauristie, K., Amm, O., Pellinen, R., Opgenoorth, H., and Pirjola, R.: STARE velocities: 2. Evening westward electron flow, Ann. Geophys., 22, 1077-1091, 2004, http://www.ann-geophys.net/22/1077/2004/.

Woodman, R. F. and Chau, J. L.: First Jicamarca radar observations of two-stream $E$ region irregularities under counter electrojet conditions, J. Geophys. Res., 107, 1482, doi:10.1029/2002JA009362, 2002. 